Chapter 5

\title{
Strategies to Decrease Morbidity After Hepatectomy for Hepatocellular Carcinoma
}

\author{
Hiroshi Sadamori, Takahito Yagi and \\ Toshiyoshi Fujiwara \\ Additional information is available at the end of the chapter \\ http://dx.doi.org/10.5772/51765
}

\section{Introduction}

In-hospital mortality rates after hepatectomy for HCC have been greatly improved due to advances in surgical techniques and perioperative management [1-4]. However, relatively high morbidity rates remain problematic, and bile leakage and organ/space surgical site infection (SSI) are still common causes of major morbidity after hepatectomy for HCC [5-13].

Various types of hepatectomy in many centres have recently been performed based on the degree of hepatic functional reserve and the location of the HCC. Anatomic hepatectomy for HCC, including subsegmentectomy, reportedly contributes to the prognosis for patients with HCC [14-16]. In addition, the rate of repeat hepatectomy for recurrent HCC has recently increased from $10 \%$ to $31 \%$ as the prognosis for patients with HCC has improved [17-22].

In our institution, anatomic and repeat hepatectomies for HCC have been performed aggressively $[12,16,22]$. We investigated risk factors for bile leakage and organ/space SSI following hepatectomies for HCC in the present series, which included a large number of patients with a high proportion of anatomic or repeat hepatectomy. Furthermore, causes, management and outcomes of intractable bile leakage and organ/space SSI were investigated and strategies to reduce major morbidity were considered.

\section{Methods}

\subsection{Patients}

Medical records of 359 patients who underwent hepatectomy without biliary reconstruction for HCC in our department between January 1, 2001 and March 31, 2010 were studied retro- 
spectively. Patients comprised 292 men and 67 women, with a mean age of 65 years (range, 32-89 years). The aetiology of liver disease was hepatitis $C$ virus in 163 patients, hepatitis $B$ virus in 122 patients, both hepatitis $C$ virus and hepatitis B virus in 31 patients, and alcoholic liver disease in 16 patients. Child-Pugh class was A in 332 patients and B in 27 patients. A total of 296 patients (82.5\%) underwent anatomic hepatectomy including subsegmentectomy. Repeat hepatectomy was performed for 59 patients $(16.4 \%)$. Repeat hepatectomy was indicated when all tumours detected on preoperative imaging could be resected within the hepatic functional reserve. When recurrent HCC tumours were $2 \mathrm{~cm}$ in maximum diameter and 3 were present, percutaneous ablation therapies were selected despite the feasibility of repeat hepatectomy, depending on tumour location in the liver.

\subsection{Surgical procedure}

Laparotomy was performed through a J incision in 287 patients, a Mercedes incision in 33 patients, a midline incision in 23 patients, and a thoraco-abdominal incision in 16 patients. Preoperative cholangiography was not usually performed. Intraoperative ultrasonography was performed to determine the extent of HCC and the line of parenchymal transection. Parenchymal transection was performed using an ultrasonic dissector (Sonop 5000; Aloka, Tokyo, Japan) combined with bipolar electrocautery. Glisson's pedicles in livers dissected by the ultrasonic dissector were ligated and small pedicles were resected using metallic surgical clips. For hemihepatectomies or extended operations, hilar dissection was performed to divide the ipsilateral branches of the hepatic artery and portal vein. The hepatic duct was exposed inside the liver during parenchymal transection and was ligated or oversewn using fine non-absorbable sutures. Parenchymal transection in hemihepatectomy or extended operations was performed largely without occlusion of vascular inflow. For segmentectomies or subsegmentectomies, Glisson's pedicle was transected at the hepatic hilus and an intermittent Pringle manoeuvre was applied during parenchymal transection.

Intraoperative cholangiography was undertaken for selected patients when the integrity of the bile duct was in doubt. A bile leakage test using a cholangiography catheter was also performed for selected patients when many Glisson's pedicles were exposed in the plane of hepatic resection. In principle, two abdominal drainage tubes were systematically positioned and the method of placing the drainage tubes was changed according to the type of hepatectomy. In hemihepatectomy, one drainage tube was placed on the cut surface of the liver and another was positioned at the Winslow hiatus. In subsegmentectomy and segmentectomy, one drainage tube was placed on the cut surface of the liver and another was positioned in the right subphrenic space. From 2001 to 2005, an open drainage system was employed using 12-mm silicone Penrose drains (Kaneka, Osaka, Japan). From 2006 to 2010, a closed drainage system was used with 24-Fr BLAKE silicone drains (Johnson \& Johnson, Somerville, NJ, USA). Drains were removed when the drainage was serous and contained no bile, usually around postoperative day (POD) 5. 


\subsection{Definition of bile leakage}

Postoperative bile leakage was defined as the drainage of macroscopic bile from surgical drains for more than 7 days after surgery. Major bile leakage was defined as macroscopic bile discharge $>100 \mathrm{ml} /$ day that did not decrease from one day to the next. Minor bile leakage was defined as bile leakage that did not fulfil the definition for major bile leakage. Intractable bile leakage was defined as bile leakage requiring endoscopic retrograde biliary drainage (ERBD) or percutaneous transhepatic biliary drainage (PTBD) during postoperative management.

\subsection{Definition of SSIs}

SSIs were defined according to the National Infections Surveillance system [23]. Using these criteria, SSIs are classified as either incisional (superficial or deep) or organ/space. Criteria for superficial incisional SSI included infection occurring at the incision site within 30 days after surgery that involved only the skin and subcutaneous tissue and at least one of the following: 1) pus discharge from the incision; 2) bacteria isolated from a sample culture from the superficial incision; 3) localized pain, tenderness, swelling, redness, or heat; and 4) wound dehiscence. Criteria for deep incisional SSI included infection of the fascia or muscle related to the surgical procedure occurring within 30 days after surgery and at least one of the following: 1) pus discharge from the deep incision; 2) spontaneous dehiscence of the incision; or 3) deliberate opening of the incision when the patient displayed the previously described signs and symptoms of infection. The definition of organ/space SSI was based on postoperative findings of at least one of the following: 1) purulent drainage from a drain without macroscopic bile discharge; or 2) intra-abdominal collection of purulent fluid confirmed at the time of reoperation or percutaneous drainage. If intra-abdominal collection at the time of reoperation or percutaneous drainage contained macroscopic bile discharge, bile leakage was considered present. If purulent fluid was drained first and macroscopic bile leakage subsequently became apparent, this was defined as bile leakage. In contrast, if drainage of purulent fluid was still observed after the cessation of macroscopic bile leakage, this was defined as organ/space SSI.

\subsection{Antimicrobial prophylaxis}

Prophylactic antibiotics regimens were as follows. With initial hepatectomy, a first-generation cephalosporin was injected intravenously within $30 \mathrm{~min}$ prior to skin incision. In patients who underwent operations lasting longer than $3 \mathrm{~h}$, additional antimicrobial agents were injected intravenously every $3 \mathrm{~h}$, as recommended by the Center for Disease Control guidelines [23]. These agents were also administered up to POD 2. In repeat hepatectomy, second-generation cephalosporin was injected intravenously in the same manner as in the initial hepatectomy and continued until POD 3.

\subsection{Intervention for methicillin-resistant Staphylococcus aureus (MRSA)}

With the exception of two emergency cases, all patients underwent preoperative evaluation for MRSA, including nasal culture. As a result, 9 of the 359 patients (2.5\%) showed 
colonisation with MRSA on admission to our institution. In those 9 patients with detection of MRSA colonisation from preoperative nasal cultures, decolonisation was performed using intranasal mupirocin therapy (administered twice daily for 3-5 days preoperatively). Prophylactic intravenous infusion of vancomycin was not applied in the 9 patients with intranasal MRSA colonisation.

\subsection{Analysis of risk factors for bile leakage and SSIs}

Patient demographics, operative and tumour factors, and preoperative liver function were evaluated to determine impacts on the occurrence of bile leakage and organ/space SSI. Preoperative factors included patient age, sex, aetiology of liver disease, Child-Pugh classification, indocyanine green dye retention rate at $15 \mathrm{~min}$ (ICG-R15), serum albumin, history of diabetes mellitus, previous radiofrequency ablation (RFA) and previous transarterial chemoembolisation (TACE). The cut-off level for ICG-R15 was set at 20\%, because ICG-R15 $<20 \%$ has been reported as the safe range for bisegmentectomy $[3,5,9]$. Surgical factors were evaluated for the type of skin incision, type of hepatectomy, number of hepatectomies, blood loss, operative time, blood transfusion, and method of abdominal drainage. With regard to the type of hepatectomy, anterior segmentectomies and medial (S4) segmentectomies were sub-grouped for analysis. The cut-off point for operative time was determined by an analysis of the receiver operating characteristics curve for bile leakage. The optimal cutoff for operative time was $306 \mathrm{~min}$; sensitivity and specificity were 0.696 and 0.728 , respectively. We thus set $300 \mathrm{~min}$ as the cut-off level for operative time. Tumour factors included the number of HCC lesions and the maximum diameter of HCC. Cut-off level for HCC diameter was determined according to results from previous reports that analysed risk factors for morbidity after hepatectomy for HCC $[3,5,9,12]$.

\subsection{Investigation of intractable bile leakage}

Management and outcomes were investigated for 46 patients with postoperative bile leakage. Indications for ERBD to treat postoperative bile leakage were based on postoperative findings of at least one of the following: 1) amount of macroscopic bile discharge from surgical drains $>200 \mathrm{ml}$ /day at 2 weeks after surgery; 2) amount of macroscopic bile discharge from surgical drains $>100 \mathrm{ml}$ /day at 4 weeks after surgery; or 3) macroscopic bile discharge from surgical drains still continuing at 6 weeks after surgery. PTBD was indicated when postoperative cholangiography and biliary drainage by ERBD were considered impractical. Intractable bile leakage necessitating ERBD or PTBD was encountered in 8 patients. The operative procedure, number of hepatectomies, timing of biliary procedures, sites of bile leakage and possible causes of bile leakage were evaluated in these 8 patients with intractable bile leakage.

\subsection{Investigation of characteristics in organ/space SSI}

Organ/space SSI was classified according to the modified Clavien system [24]: grade I, minor risk events not requiring special treatment; grade II, potentially life-threatening complications requiring pharmacological treatment; grade III, complications requiring surgical, endoscopic or radiological intervention, either with (III-b) or without (III-a) general anaesthesia; grade IV, 
life-threatening complications involving dysfunction of one (IV-a) or multiple (IV-b) major organs; and grade V, complications resulting in the death of the patient. Management and outcomes were investigated for 31 patients with organ/space SSI. In addition, the causative bacterium was identified for both incisional and organ/space SSIs. Furthermore, pre- and intraoperative parameters, causative bacteria and hospitalisation were compared between groups classified by the number of hepatectomies in patients with organ/space SSI.

\subsection{Statistical analysis}

Operative time, blood loss and postoperative hospital stay are presented as mean \pm standard error of the mean. Differences in qualitative variables were assessed using Fisher's exact test or the ${ }^{2}$ test, while differences in quantitative variables were analysed using the Mann-Whitney test. Uni- and multivariate logistic regression analyses were used to identify risk factors for bile leakage and organ/space SSI based on the 18 above-mentioned clinical factors. Relative risk was described by the estimated odds ratio (OR) with a 95\% confidence interval. Two-sided $P$ values were computed and an effect was considered significant at the level of $P 0.05$. All statistical analyses were performed using SPSS II statistical software (SPSS, Tokyo, Japan).

\section{Results}

\subsection{Risk factors for bile leakage (Tables 1, 3)}

Univariate logistic regression analysis revealed several factors associated with increased risk of developing bile leakage. Repeat hepatectomy influenced the risk of developing bile leakage, with an OR of 3.78 compared to the initial hepatectomy. In contrast, neither previous RFA nor TACE had any significant impact on the occurrence of bile leakage. Operative time 300 min was associated with increased risk (OR, 5.32; $P<0.001)$, as was blood loss $2000 \mathrm{ml}$ $(\mathrm{OR}, 4.12 ; P<0.001)$. Multivariate analysis regarding bile leakage confirmed operative time $300 \mathrm{~min}$ as an independent risk factor.

\begin{tabular}{lccc}
\hline Variable & OR & $95 \% \mathrm{CI}$ & $P$ \\
\hline Bile leakage & & & \\
Operative time $(<300 \mathrm{~min}$ vs. $\geqq 300 \mathrm{~min})$ & 5.32 & $2.71-10.4$ & $<0.001$ \\
Blood loss $(<2000 \mathrm{ml}$ vs. $\geqq 2000 \mathrm{ml})$ & 4.12 & $2.07-3.20$ & $<0.001$ \\
Number of hepatectomies (initial vs. repeat) & 3.78 & $1.91-7.48$ & $<0.001$ \\
\hline
\end{tabular}

Table 1. Univariate analysis of risk factors for bile leakage.

\subsection{Risk factors for SSIs (Tables 2, 3)}

SSIs developed in $14.5 \%$ of patients $(n=52)$, and 3 patients showed both incisional and organ/ space SSIs. Univariate logistic regression analysis revealed several factors associated with 
increased risk of developing SSIs. Repeat hepatectomy influenced the risk of developing SSIs, with an OR of 8.27 for initial hepatectomy. Operative time $300 \mathrm{~min}$ was associated with increased risk $(\mathrm{OR}, 4.46 ; P<0.001)$. The presence of blood transfusion influenced the risk of developing SSIs. Presence of bile leakage was associated with increased risk of SSIs (OR, 6.40; $P=0.002$ ). Multivariate analysis regarding SSIs confirmed both repeat hepatectomy and operative time $300 \mathrm{~min}$ as independent risk factors.

\subsection{Risk factor for incisional SSI (Tables 2, 3)}

Incidence of incisional SSI was $6.7 \%(n=24)$. Univariate logistic regression analysis revealed that the presence of blood transfusion was associated with increased risk of developing incisional SSI. Type of skin incision classified according to the presence or absence of transverse incision showed no significant influence on the occurrence of incisional SSI in this series. Multivariate analysis regarding incisional SSI confirmed the presence of blood transfusion as an independent risk factor.

\subsection{Risk factors for organ/space SSI (Tables 2, 3)}

Organ/space SSI developed in $8.6 \%$ of patients $(n=31)$. Univariate logistic regression analysis revealed several factors associated with increased risk of developing organ/space SSI. Repeat hepatectomy influenced the risk of developing organ/space SSI, with an OR of 4.29 compared to initial hepatectomy. In contrast, neither previous RFA nor TACE exerted any significant impact on occurrence of organ/space SSI.

\begin{tabular}{llll}
\hline Variable & OR & $95 \% \mathrm{CI}$ & $P$ \\
\hline SSIs & & & \\
Operative time (<300 min vs. $\geqq 300 \mathrm{~min})$ & 4.46 & $1.64-5.46$ & $<0.001$ \\
Number of hepatectomies (initial vs. repeat) & 8.27 & $2.24-8.24$ & $<0.001$ \\
Bileleakage (absence vs. presence) & 6.40 & $1.55-6.46$ & 0.002 \\
Blood transfusion (absence vs. presence) & 2.05 & $1.37-4.55$ & 0.003 \\
Incisional SSI & & & \\
Blood transfusion (absence vs. presence) & 4.38 & $1.85-10.4$ & $<0.001$ \\
Organ/space SSI & & & \\
Number of hepatectomies (initial vs. repeat) & 4.29 & $3.79-18.0$ & $<0.001$ \\
Bileleakage (absence vs. presence) & 3.16 & $2.90-14.3$ & $<0.001$ \\
Operative time (<300 min vs. $\geqq 300$ min) & 2.99 & $2.03-9.81$ & $<0.001$ \\
Bloodloss (<2000 ml vs. $\geqq 2000$ ml) & 2.63 & $0.73-6.59$ & 0.010 \\
\hline
\end{tabular}

Table 2. Univariate analysis of risk factors for SSIs.

The method of abdominal drainage (open Penrose drains or closed suction drains) showed no significant influence. Operative time $300 \mathrm{~min}$ was associated with increased risk of organ/space SSI (OR, 2.99; $P<0.001)$. Presence of bile leakage was likewise associated with in- 
creased risk (OR, 3.16; $P=0.01)$. Blood loss $2000 \mathrm{ml}$ was associated with increased risk (OR, $2.63 ; P<0.001)$. Multivariate analysis confirmed both repeat hepatectomies and presence of bile leakage as independent risk factors for organ/space SSI.

\begin{tabular}{llll}
\hline Variable & OR & $95 \% \mathrm{CI}$ & $P$ \\
\hline $\begin{array}{l}\text { Bile leakage } \\
\text { Operative time (<300 min vs. } \geqq 300 \mathrm{~min})\end{array}$ & 25.9 & $2.28-29.4$ & 0.009 \\
SSIs & & & \\
Number of hepatectomies (initial vs. repeat) & 3.43 & $1.73-6.80$ & $<0.001$ \\
Operative time (<280 min vs. $\geqq 280$ min) & 2.32 & $1.22-4.43$ & 0.011 \\
Incisional SSI & & & \\
$\quad$ Blood transfusion (absence vs. presence) & 7.56 & $2.58-22.1<0.001$ \\
$\begin{array}{l}\text { Organ/space SSI } \\
\text { Number of hepatectomies (initial vs. repeat) }\end{array}$ & 6.15 & $2.69-14.1<0.001$ \\
Bile leakage (absence vs. presence) & 3.01 & $1.20-7.56$ & 0.018 \\
\hline
\end{tabular}

Table 3. Multivariate analysis of risk factors for bile leakage and SSIs.

\subsection{Management and outcomes of bile leakage (Figure 1)}

Management and outcomes of the 46 patients with bile leakage are shown in Figure 1.

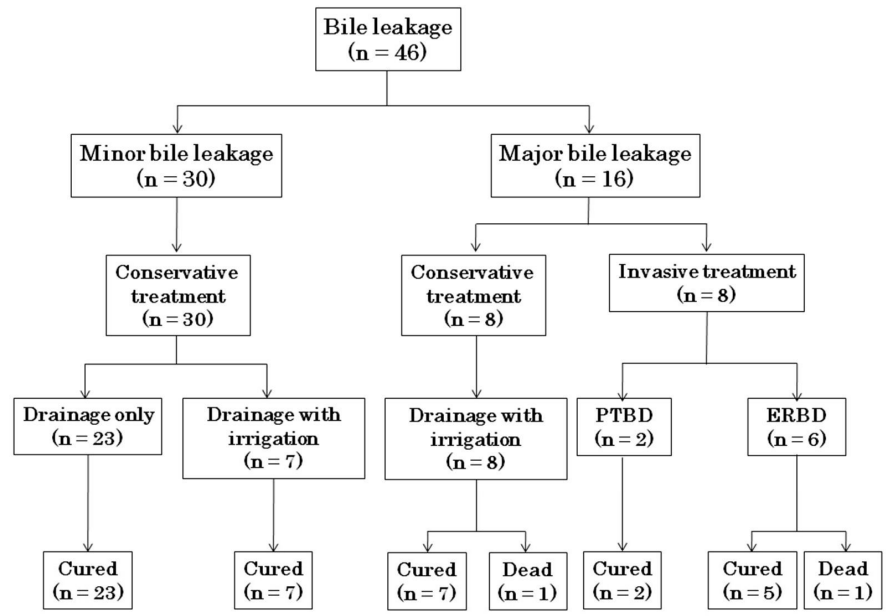

Figure 1. Medical management and outcomes for patients with postoperative bile leakage.

Minor bile leakage in 30 patients (65\%) was controllable and cured by conservative therapies comprising drainage alone in 23 patients and drainage with irrigation in 7 patients. Sixteen patients (35\%) showed complications of major bile leakage. In 8 of these patients, the major bile 
leakage was treated using drainage with irrigation. One patient died due to subsequent intractable ascites and liver failure during drainage with irrigation, while the other 7 patients healed. The remaining 8 patients with major bile leakage needed either ERBD or PTBD.

\subsection{Characteristics of 8 patients with intractable bile leakage (Table 4)}

We investigated the characteristics of the 8 patients who needed either ERBD or PTBD for bile leakage. High-risk surgical procedures were performed in most of these cases and repeat hepatectomy was performed in 6 of the 8 patients. The median timing of biliary procedures was POD 21.5 (range, POD 2-45). Bile leakage sites identified on postoperative cholangiography included the hepatic duct in 2 patients and the raw surface of the liver in 6 patients. Possible causes of bile leakage as assessed by postoperative cholangiography were as follows: stricture of the hepatic duct that existed preoperatively, possibly due to previous treatments for HCC in 4 patients ( 2 patients due to previous hepatectomies, 1 patient due to previous TACE, 1 patient due to previous RFA), stricture of the hepato-jejunostomy from previous pancreatoduodenectomy in 1 patient, dyskinesis of the papilla of Vater in 1 patient and intraoperative injury of the left hepatic duct related to repeat hepatectomy in 2 patients. Three of these 8 patients subsequently showed complications of intractable ascites. In 2 patients, both bile leakage and intractable ascites were cured without intra-abdominal septic complications. The other patient with stricture and injury of the left hepatic duct caused by a previous RFA died due to intractable ascites, uncontrollable biliary infection and liver failure. Bile leakage in the other 5 patients healed after either ERBD or PTBD, with no other major morbidities.

\begin{tabular}{|c|c|c|c|c|c|c|}
\hline Age/Sex & $\begin{array}{l}\text { Operative } \\
\text { procedure he }\end{array}$ & $\begin{array}{l}\text { Number of } \\
\text { hepatectomies }\end{array}$ & $\begin{array}{c}\text { Biliary } \\
\text { procedure } \\
\text { (Timing) }\end{array}$ & $\begin{array}{l}\text { Site of } \\
\text { bile leakage }\end{array}$ & Cause of bile leakage & Outcome \\
\hline $62 / \mathrm{M}$ & Caudate lob. & Repeat & $\begin{array}{l}\text { Endoscopic } \\
\text { (14POD) }\end{array}$ & Lt. hepatic duct & $\begin{array}{l}\text { Stricture andinjury of lt hepatic } \\
\text { duct due toprevious RFA }\end{array}$ & $\begin{array}{c}\text { Died } \\
\text { (Biliary infection } \\
\text { and liver failure) }\end{array}$ \\
\hline $53 / \mathrm{M}$ & Anterior seg. & Repeat & $\begin{array}{l}\text { Endoscopic } \\
\text { (10POD) }\end{array}$ & Lt. hepatic duct & $\begin{array}{l}\text { Intra-operative injury of } \\
\text { lt.hepatic duct }\end{array}$ & Cured \\
\hline $72 / \mathrm{M}$ & S5 subseg. & Initial & $\begin{array}{l}\text { PTBD } \\
\text { (43POD) }\end{array}$ & $\begin{array}{l}\text { Raw surface } \\
\text { of liver }\end{array}$ & $\begin{array}{l}\text { Stricture of hepato jejunostomy of } \\
\text { previous pancreatoduodenectomy }\end{array}$ & Cured \\
\hline $60 / \mathrm{M}$ & S6 subseg. & Repeat & $\begin{array}{l}\text { Endosopic } \\
\text { (30POD) }\end{array}$ & $\begin{array}{l}\text { Raw surface } \\
\text { of liver }\end{array}$ & $\begin{array}{l}\text { Stricture of rt. hepatic duct due to } \\
\text { previous hepatectomy }\end{array}$ & Cured \\
\hline $62 / \mathrm{M}$ & S8 partial hep. & . Initial & $\begin{array}{l}\text { Endosopic } \\
\text { (2POD) }\end{array}$ & $\begin{array}{l}\text { Raw surface } \\
\text { of liver }\end{array}$ & $\begin{array}{l}\text { Dyskinesis of the papilla } \\
\text { of Vater }\end{array}$ & Cured \\
\hline $67 / \mathrm{F}$ & Central biseg. & Repeat & $\begin{array}{l}\text { PTBD } \\
\text { (45POD) }\end{array}$ & $\begin{array}{l}\text { Raw surface } \\
\text { of liver }\end{array}$ & $\begin{array}{l}\text { Stricture of rthepatic duct due to } \\
\text { in tra-operative injury }\end{array}$ & Cured \\
\hline $50 / \mathrm{M}$ & Posterior seg. & Repeat & $\begin{array}{l}\text { Endoscopic } \\
\text { (15POD) }\end{array}$ & $\begin{array}{l}\text { Raw surface } \\
\text { of liver }\end{array}$ & $\begin{array}{l}\text { Stricture of rt. hepatic duct due to } \\
\text { previous TACE }\end{array}$ & Cured \\
\hline $70 / \mathrm{F}$ & S4 seg. & Repeat & $\begin{array}{l}\text { Endoscopic } \\
\text { (28POD) }\end{array}$ & $\begin{array}{l}\text { Raw surface } \\
\text { of liver }\end{array}$ & $\begin{array}{l}\text { Stricture of lt hepatic duct due to } \\
\text { previous hepatectomy }\end{array}$ & Cured \\
\hline
\end{tabular}

lob = lobectomy; seg = segmentectomy; hep = hepatectomy; PTBD = percutaneous transhepatic biliary drainage: $\mathrm{RFA}=$ radiofrequency ablation $\mathrm{TACE}=$ transcatheter arterial chemoembolization; $\mathrm{POD}=$ postoperative day

Table 4. Characteristic and management of 8 patients with intractable bile leakage. 


\subsection{Management and outcome of organ/space SSI}

Organ/space SSI in 31 patients was classified as follows: abscess on the cut surface of the liver in 26 patients; right subphrenic abscess in 4 patients; and liver abscess in 1 patient. One of the 31 patients with organ/space SSI was treated by reoperation due to right subphrenic abscess, but died due to myocardial infarction. Eleven patients needed percutaneous drainage of organ/space SSI and all of them were cured. Organ/space SSI in 19 patients healed with irrigation of the pre-existing drain. As a result, 31 patients with organ/space SSI were stratified according to the modified Clavien system as follows: grade I, 0 patients; II, 13 patients; III-a, 15 patients; III-b, 2 patients; IV-a, 1 patient; IV-b, 0 patients; and V, 0 patients. No mortality was associated with organ/space SSI in this series, but the postoperative hospital stay was significantly longer for patients with organ/space SSI (53 7.2 days) than for patients without organ/space SSI (27 0.9 days, $\mathrm{P}=0.001)$.

\subsection{Bacteria causing incisional and organ/space SSI (Table 5)}

Causative bacteria for incisional and organ/space SSI comprised gram-positive cocci in 17 patients $(70.8 \%)$ and 19 patients (61.3\%), and gram-negative rods in 6 patients $(25.0 \%)$ and 9 patients $(29.0 \%)$, respectively, indicating similar proportions of gram-positive cocci and gram-negative rods in both incisional and organ/space SSI. MRSA was the causative bacteria in 12 of 19 patients with organ/space SSI caused by gram-positive cocci.

\begin{tabular}{lll}
\hline Causative bacteria & $\begin{array}{c}\text { Incisional } \\
(\mathbf{n}=\mathbf{2 4})\end{array}$ & $\begin{array}{c}\text { Organ/space } \\
(\mathbf{n}=31)\end{array}$ \\
\hline Gram-positive cocci & & \\
MRSA & 8 & 12 \\
MSSA & 0 & 1 \\
S.epidermidis & 4 & 2 \\
Enterococcus sp. & 4 & 4 \\
Steptococcus sp. & 1 & 0 \\
\hline Total & $17(70.8 \%)$ & $19(61.3 \%)$ \\
\hline Gram-negative bacilli & & \\
Escherichia coli & 1 & 2 \\
Klebsiella sp. & 2 & 1 \\
Pseudomonas sp. & 2 & 4 \\
Enterobacter sp. & 1 & 1 \\
Bacteroides sp. & 0 & $9(29.0 \%)$ \\
\hline Total & $6(25.0 \%)$ & 3 \\
\hline Negative & 1 & \\
\hline
\end{tabular}

MRSA: Methicillin-resistant Staphylococcus aureus

MSSA : Methicillin-sensitive Staphylococcus aureus

Table 5. Causative bacteria of incisional and organ/space SSI. 


\subsection{Comparison between initial and repeat hepatectomies in patients with organ/space} SSI (Table 6)

We compared clinical parameters between initial and repeat hepatectomies in patients with organ/space SSI (Table 6). HCC diameter was significantly larger in patients with organ/ space SSI who underwent initial hepatectomy than in patients who underwent repeat hepatectomy. No significant differences were seen between groups in any other preoperative parameters, including patient demographics and preoperative liver function. No significant differences were identified between groups in operative parameters, including blood loss, operative time and blood transfusion. Rates of bile leakage were similar between groups. In contrast, in terms of bacteria causing organ/space SSI, detection of MRSA was significantly more frequent in the repeat hepatectomy group than in the initial group.

\begin{tabular}{|c|c|c|c|}
\hline & \multicolumn{2}{|c|}{ Number of hepatectomies } & \multirow[b]{2}{*}{ Pvalue } \\
\hline & Initial (n=14) & Repeat $(n=17)$ & \\
\hline Age & $59.6 \pm 3.2$ & $62.2 \pm 2.5$ & 0.523 \\
\hline \multicolumn{4}{|l|}{ Etiology of liver disease } \\
\hline HCV-related & 5 & 5 & 0.713 \\
\hline HBV-related & 6 & 11 & 0.231 \\
\hline HCV+HBV & 2 & 0 & 0.113 \\
\hline \multicolumn{4}{|l|}{ Child-Pugh class } \\
\hline $\mathrm{A} / \mathrm{B}$ & $14 / 0$ & $15 / 2$ & 0.192 \\
\hline ICGR15(\%) & $12.7 \pm 1.7$ & $18.5 \pm 3.4$ & 0.149 \\
\hline Albumin (g/dl) & $4.0 \pm 0.1$ & $3.9 \pm 0.1$ & 0.610 \\
\hline \multicolumn{4}{|l|}{ Diabetes mellitus } \\
\hline Negative/Positive & 1212 & $13 / 4$ & 0.524 \\
\hline \multicolumn{4}{|l|}{ Number of HCC lesions } \\
\hline $1 />1$ & $11 / 3$ & $8 / 9$ & 0.078 \\
\hline Diameter of $\mathrm{HCC}(\mathrm{cm})$ & $4.5 \pm 0.9$ & $2.4 \pm 0.3$ & $<0.05$ \\
\hline \multicolumn{4}{|l|}{ Type of hepatectomy } \\
\hline Partial hepatectomy & 0 & 3 & 0.104 \\
\hline Subsegmentectomy & 3 & 5 & 0.619 \\
\hline Segmentectomy & 4 & 5 & 0.960 \\
\hline Hemihepatectomy & 7 & 4 & 0.132 \\
\hline Trisegmentectomy & 0 & 0 & \\
\hline Bloodloss (ml) & $1833 \pm 511$ & $1697 \pm 307$ & 0.822 \\
\hline Operative time (min) & $333 \pm 11$ & $343 \pm 29$ & 0.767 \\
\hline \multicolumn{4}{|l|}{ Blood transfusion } \\
\hline Absence/Presence & $9 / 5$ & $8 / 9$ & 0.345 \\
\hline \multicolumn{4}{|l|}{ Bileleakage } \\
\hline Absence/Presence & $9 / 5$ & $10 / 7$ & 0.531 \\
\hline \multicolumn{4}{|l|}{ MRSA } \\
\hline Negative/Positive & $12 / 2$ & $7 / 10$ & $<0.05$ \\
\hline Hospital stay(days) & $41 \pm 7$ & $63 \pm 11$ & 0.111 \\
\hline
\end{tabular}

HCV: hepatitis $C$ virus, HBV. hepatitis $B$ virus, ICG $R-15$ in docyanine green dye retension rate at $15 \mathrm{~min}, \mathrm{MRSA}$ : Methicillin-resistant $\mathrm{S}$ taphylococcus aureus

Table 6. Comparison between initial nad repeat hepatectomies in patients with organ/space SSI. 


\section{Discussion}

In-hospital mortality rates after hepatectomy for HCC have been greatly improved due to advances in surgical techniques and perioperative management [1-4]. However, relatively high morbidity rates remain problematic. The overall morbidity rates after hepatectomy for liver tumors have been reported to be $22.6-47.7 \%$, and bile leakage and organ/space surgical site infection (SSI) are still common causes of major morbidity after hepatectomy for HCC [5-13]. Various types of hepatectomy in many centres have recently been performed based on the degree of hepatic functional reserve and the location of the HCC. In addition, the rate of repeat hepatectomy for recurrent HCC has recently increased from $10 \%$ to $31 \%$ as the prognosis for patients with HCC has improved [17-22]. The characteristic of our study is that this series consisted of a large number and percentage of both anatomic and repeat hepatectomies for HCC.

Rates of bile leakage after hepatectomy for liver tumours and benign lesions have been reported as 3.6\%-12.0\%, varying widely among different studies $[6,7,11,12,25-30]$. However, no standardised definition of bile leakage after hepatectomy has been established. In previous reports $[6,8,11,13,30]$, the definition based on the drainage of macroscopic bile has been adopted. Several studies has proposed the definition on quantitative basis using the bilirubin concentration within the drain [26, 28], but these cut-off values varied. Currently, the International Study Group of Liver Surgery has proposed a consensus definition of bile leakage based on the postoperative course of bilirubin concentration in serum and drainage fluid [31]. Application of a uniform definition of bile leakage is indispensable to enabling standardised comparison of the results of different clinical reports and to facilitating objective evaluation of therapeutic modalities in the field of hepatectomies.

In the present study, prolonged operative time was identified as an independent risk factor for bile leakage and the type of hepatectomy had no significant impact on the rate of bile leakage. Several groups have reported that hepatectomies in which the cut surface exposed the major Glisson's sheath (i.e., central bisegmentectomy, S4 segmentectomy, and S8 subsegmentectomy) were independent risk factors for bile leakage [8, 28-30]. However, our results indicate that the standard types of hepatectomy were not risk factors for bile leakage, even if a wide cut surface with an exposed major Glisson's sheath was necessary, when assessment of liver function was appropriate and surgical procedures were performed carefully during transection of the liver parenchyma. We assume that the prolongation of operative time in this study was related to the extended duration of liver parenchymal transection and/or resection for severe intra-abdominal adhesions around the liver.

Our results revealed latent stricture of the biliary anatomy and intraoperative injury of the hepatic duct related to repeat hepatectomy as the main causes of intractable bile leakage requiring invasive treatment. Preoperative assessment of the biliary anatomy should therefore be considered for selected patients at high risk of intractable bile leakage. Various measures could also be applied during surgery to diminish the incidence of major and intractable bile leakage. First, intraoperative cholangiography should be used, particularly in repeat hepatectomies and in patients who have been treated with RFA or TACE for HCC located in the 
hepatic hilar region, as the identification of bile duct injury or stricture could allow immediate correction. Second, T-tube drainage or trans-cystic duct drainage of the common bile duct could be indicated in patients needing decompression of the biliary tree, such as patients with dyskinesis of the papilla of Vater. Third, particularly in repeat systematised hepatectomies, division of the bile ducts could be performed inside the liver during parenchymal transection, as this procedure could decrease the risk of injury to the bile ducts compared to division of the bile ducts at the liver hilum.

In the 1980s and 1990s, organ/space SSI formation after hepatectomy was reported as a fatal complication causing liver failure and death [32-34]. Although rates of organ/space SSI after hepatectomy have been reported as 4.7\%-25\% [35-42], hospital mortality rates caused by organ/space SSI have declined [7-10, 36, 40]. Several groups have reported high patient age and presence of diabetes mellitus as independent risk factors for organ/space SSI [36, 39]. However, these variables were not identified as independent risk factors for organ/space SSI in the present study. Our key result was the identification of repeat hepatectomy as an independent risk factor for organ/space SSI, suggesting that treatment strategies need to be established to reduce the high rate of organ/space SSI after repeat hepatectomy.

Repeat hepatectomy was identified as an independent risk factor for SSI and organ/space SSI, but previous RFA and TACE were not. Repeat hepatectomy for recurrent HCC is useful in establishing the good long-term outcomes. Cumulative 5-year survival rates after second hepatectomy have been reported as $41-69 \%$ [17-22]. RFA has recently been confirmed as a safe and promising therapy for recurrent HCC after hepatectomy. However, sufficient evidence does not exist to confirm whether RFA actually improves long-term outcomes. Cumulative 5-year survival rates after RFA for recurrent HCC after hepatectomy have been reported as 18-51.6\% [43-45]. RFA is sometimes ineffective for HCC on the liver surface or near large vessels. In addition, postoperative adhesions between the remnant liver and gastrointestinal tract may prevent safe percutaneous RFA in patients with recurrent HCC.

In this study, MRSA was detected more frequently in organ/space SSI after repeat hepatectomy compared with after initial hepatectomy. We assume that most organ/space SSIs with MRSA after repeat hepatectomy develop as a result of contamination when the surgical procedure comes into contact with intra-abdominal colonisation or micro-abscesses of MRSA that had formed after the initial hepatectomy. This assumption might be partially supported by our result that the method of abdominal drainage (open or closed) had no significant influence on the occurrence of organ/space SSI. If this assumption is valid, preoperative interventions for MRSA, consisting of nasal culture and decolonisation of nasal MRSA, will not greatly reduce the occurrence of organ/space SSI involving MRSA after repeat hepatectomy. Walsh et al. recently reported that an MRSA intervention program, in which all patients received intranasal mupirocin and those patients colonised with MRSA received prophylactic intravenous infusion of vancomycin, resulted in nearcomplete and sustained elimination of MRSA SSIs after cardiac surgery [46]. Regarding patients who undergo repeat hepatectomies, preoperative detection of intra-abdominal colonisation or micro-abscess containing MRSA is difficult. MRSA intervention programs 
thus need to be improved, particularly for patients who undergo repeat hepatectomies, by considering the prophylactic intravenous administration of vancomycin.

In conclusion, our results reveal prolonged operative time as an independent risk factor for bile leakage, and latent stricture of the biliary anatomy and intraoperative injury of the hepatic duct related to repeat hepatectomy as the main causes of intractable bile leakage necessitating invasive treatment. Repeat hepatectomy was also identified as an independent risk factor for organ/space SSI, with MRSA as the main causative bacteria in organ/space SSI after repeat hepatectomy for HCC. Establishment of treatment strategies is thus important for reducing the high rate of organ/space SSI after repeat hepatectomy. In addition, preoperative assessment of the biliary anatomy and surgical procedures to decrease the incidence of major bile leakage should be considered for selected patients at high risk of intractable bile leakage.

\section{Author details}

Hiroshi Sadamori*, Takahito Yagi and Toshiyoshi Fujiwara

*Address all correspondence to: sada@md.okayama-u.ac.jp

Department of Gastroenterological Surgery, Okayama University Graduate School of Medicine, Dentistry and Pharmaceutical Sciences, Okayama, Japan

\section{References}

[1] Fan, S. T., Lo, C. M., Liu, C. L., et al. (1999). Hepatectomy for hepatocellular carcinoma: toward zero hospital deaths. Ann Surg, 229, 323-330.

[2] Fong, Y., Sun, R. L., Jarnagin, W., \& Blumgart, L. H. (1999). An analysis of 412 cases of hepatocellular carcinoma at a Western center. Ann Surg, 229, 790-800.

[3] Torzilli, G., Makuuchi, M., Inoue, K., et al. (2007). No mortality liver resection for hepatocellular carcinoma in cirrhotic and noncirrhotic patients. Arch Surg, 134, 984-992.

[4] Sadamori, H., Yagi, T., Matsuda, H., et al. (2010). Risk factors for major morbidity after hepatectomy for hepatocellular carcinoma in 293 recent cases. J Hepatobiliary Pancreat Sci, 17, 709-718.

[5] Shimada, M., Takenaka, K., Fujiwara, Y., et al. (1998). Risk factors linked to postoperative morbidity in patients with hepatocellular carcinoma. Br J Surg, 85, 195-198.

[6] Lo, C. M., Fan, S. T., Liu, C. L., Lai, E. C. S., \& Wong, J. (1998). Biliary complication after hepatic resection-Risk factors, management, and outcome. Arch Surg, 133, 156-161. 
[7] Belghiti, J., Hiramatsu, K., Benoist, S., Massault, P., Sauvanet, A., \& Farges, O. (2000). Seven hundred forty-seven hepatectomies in the 1990s: an update to evaluate the actual risk of liver resection. J Am Coll Surg, 191, 38-46.

[8] Yamashita, Y., Hamatsu, T., Rikimaru, T., et al. (2001). Bile leakage after hepatic resection. Ann Surg, 233, 45-50.

[9] Capussotti, L., Muratore, A., Amisano, M., Polastri, R., Bouzari, H., \& Massucco, P. (2005). Liver resection for hepatocellular carcinoma on cirrhosis: analysis of mortality, morbidity and survival-a European single center experience. Eur J Surg Oncol, 31, 986-993.

[10] Taketomi, A., Kitagawa, D., Itoh, S., et al. (2007). Trends in morbidity and mortality after hepatic resection for hepatocellular carcinoma: An institute's experience with 625 patients. J Am Coll Surg, 204, 580-587.

[11] Virani, S., Michaelson, J., Hutter, M., et al. (2007). Morbidity and mortality after liver resection: Results of the patient safety in surgery study. J Am Coll Surg, 204, 1284-1292.

[12] Sadamori, H., Yagi, T., Shinoura, S., et al. (2012). Risk factors of organ/space surgical site infection after hepatectomy for hepatocellular carcinoma in 359 recent cases. J Hepatobiliary Pancreat Sci, Jan 25. [Epub ahead of print]. PMID: 22273719.

[13] Sadamori, H., Yagi, T., Shinoura, S., et al. (2012). Intractable bile leakage after hepatectomy for hepatocellular carcinoma in 359 recent cases. Dig Surg, 29, 149-156.

[14] Hasegawa, K., Kokudo, N., Imamura, H., et al. (2005). Prognostic impact of anatomic resection for hepatocellular carcinoma. Ann Surg, 242, 252-259.

[15] Eguchi, S., Kanematsu, T., Arii, S., et al. (2008). Liver Cancer Study Group of Japan. Comparison of the outcomes between an anatomical subsegmentectomy and a nonanatomical minor hepatectomy for single hepatocellular carcinomas based on a Japanese nationwide survey. Surgery, 143, 469-475.

[16] Sadamori, H., Matsuda, H., Shinoura, S., et al. (2009). Anatomical subsegmentectomy in the lateral segment for hepatocellular carcinoma. Hepatogastroenterology, 56, 971-977.

[17] Farges, O., Regimbeau, J. M., \& Belghiti, J. (1998). Aggressive management of recurrence following surgical resection of hepatocellular carcinoma. Hepatogastroenterolo$g y, 45,1275-1280$.

[18] Shimada, M., Takenaka, K., Taguchi, K., et al. (1998). Prognostic factors after repeat hepatectomy for recurrent hepatocellular carcinoma. Ann Surg, 227, 80-85.

[19] Poon, R. T., Fan, S. T., Lo, C. M., Liu, C. L., \& Wong, J. (1999). Intrahepatic recurrence after curative resection of hepatocellular carcinoma: long-term results of treatment and prognostic factors. Ann Surg, 229, 216-222. 
[20] Minagawa, M., Makuuchi, M., Takayama, T., \& Kokudo, N. (2003). Selection criteria for repeat hepatectomy in patients with recurrence hepatocellular carcinoma. Ann Surg, 238, 703-710.

[21] Itamoto, T., Nakahara, H., Amano, H., et al. (2007). Repeat hepatectomy for recurrent hepatocellular carcinoma. Surgery, 141, 589-597.

[22] Umeda, Y., Matsuda, H., Sadamori, H., Matsukawa, H., Yagi, T., \& Fujiwara, T. (2011). A prognostic model and treatment strategy for intrahepatic recurrence of hepatocellular carcinoma after curative resection. World J Surg, 35, 170-177.

[23] CDC NNIS System. (2004). National Infections Surveillance (NNIS) system report, data summary from January 1992 to June 2004, issued October 2004. Am J Infect Control, 32, 470-485.

[24] Dindo, D., Demartines, N., \& Clavien, P. A. (2004). Classification of surgical complications: a new proposal with evaluation in a cohort of 6336 patients and results of a survey. Ann Surg, 240, 205-213.

[25] Benzoni, E., Cojutti, A., Lorenzin, D., et al. (2007). Liver resective surgery: a multivariate analysis of postoperative outcome and complication. Langenbecks Arch Surg, $392,45-54$.

[26] Tanaka, S., Hirohashi, K., Tanaka, H., et al. (2002). Incidence and management of bile leakage after hepatic resection for malignant hepatic tumors. J Am Coll Surg, 195, 484-489.

[27] Reed, D. N., Jr Vitale, G. C., Wrightson, W. R., Edwards, M., \& Mc Masters, K. (2003). Decreasing mortality of bile leaks after elective hepatic surgery. Am J Surg, 185, 316-318.

[28] Nagano, Y., Togo, S., Tanaka, K., et al. (2003). Risk factors and management of bile leakage after hepatic resection. World J Surg, 27, 695-698.

[29] Lee, C. C., Chau, G. Y., Lui, W. Y., et al. (2005). Risk factors associated with bile leakage after hepatic resection for hepatocellular carcinoma. Hepatogastroenterology, 52, 1168-1171.

[30] Capussotti, L., Ferrero, A., Vigano, L., Sgotto, E., Muratore, A., \& Polastri, R. (2006). Bile leakage and liver resection: Where in the risk? Langenbecks Arch Surg, 141, 690-694.

[31] Koch, M., Garden, O. J., Padbury, R., et al. (2011). Bile leakage after hepatobiliary and pancreatic surgery: A definition and grading of severity by the International Study Group of Liver Surgery. Surgery, 149, 680-688.

[32] Yanaga, K., Kanematsu, T., Takenaka, K., \& Sugimachi, K. (1986). Intraperitoneal septic complications after hepatectomy. Ann Surg, 203, 148-152.

[33] Anderson, R., Saarela, A., Tranberg, K. G., \& Bengmark, S. (1990). Intraabdominal abscess formation after major liver resection. Acta Chir Scand, 156, 707-710. 
[34] Nagasue, N., Kohno, H., Tachibana, M., Yamanoi, A., Ohmori, H., \& El-Assai, O. (1999). Prognostic factors after hepatic resection for hepatocellular carcinoma associated with Child-Turcotte class B and C cirrhosis. Ann Surg, 229, 84-90.

[35] Wu, C. C., Yeh, D. C., Lin, M. C., Liu, T. J., \& P'eng, F. K. (1998). Prospective randomized trial of systemic antibiotics in patients undergoing liver resection. Br J Surg, 85, 489-493.

[36] Togo, S., Matsuo, K., Tanaka, K., et al. (2007). Perioperative infection control and its effectiveness in hepatectomy. J Gastroenterol Hepatol, 22, 1942-1948.

[37] Shiba, H., Ishii, Y., Ishida, Y., et al. (2009). Assessment of blood-products use as predictor of pulmonary complications and surgical infection after hepatectomy for hepatocellular carcinoma. J Hepatobiliary Pancreat Surg, 16, 69-74.

[38] Okabayashi, T., Nishimori, I., Yamashita, K., et al. (2009). Risk factors and predictors for surgical site infection after hepatic resection. J Hospital Infect, 73, 47-53.

[39] Kobayashi, S., Gotohda, N., Nakagohri, T., Takahashi, S., Konishi, M., \& Kinoshita, T. (2009). Risk factors of surgical site infection after hepatectomy for liver cancers. World J Surg, 33, 312-317.

[40] Uchiyama, K., Ueno, M., Ozawa, S., et al. (2011). Risk factors for postoperative infectious complications after hepatectomy. J Hepatobiliary Pancreat Sci, 18, 67-73.

[41] Togo, S., Kubota, T., Takahashi, T., et al. (2008). Usefulness of absorbable sutures in preventing surgical site infection in hepatectomy. J Gastrointest Surg, 12, 1041-1046.

[42] Arikawa, T., Kurokawa, T., Ohwa, Y., et al. (2011). Risk factors for surgical site infection after hepatectomy for hepatocellular carcinoma. Hepatogastroenterology, 58, 143-146.

[43] Lau, W. Y., \& Lai, E. C. (2009). The current role of radiofrequency ablation in the management of hepatocellular carcinoma: a systemic review. Ann Surg, 249, 20-5.

[44] Choi, D., Lim, H. K., Rhim, H., Kim, Y. S., Yoo, B. C., Paik, S. W., et al. (2007). Percutaneous radiofrequency ablation for recurrent hepatocellular carcinoma after hepatectomy: long-term results and prognostic factors. Ann Surg Oncol, 14, 2319-9.

[45] Taura, K., Ikai, I., Hatano, E., Fujii, H., Uyama, N., \& Shimahara, Y. (2006). Implication of frequent local ablation therapy for intrahepatic recurrence in prolonged survival of patients with hepatocellular carcinoma undergoing hepatic resection: an analysis of 610 patients over 16 years old. Ann Surg, 244, 265-73.

[46] Walsh, E. E., Greene, L., \& Kirshner, R. (2011). Sustained reduction in methicillin-resistant Staphylococcus aureus wound infections after cardiothoracic surgery. Arch Intern Med, 171, 68-73. 